University of Puget Sound

Sound Ideas

All Faculty Scholarship

Faculty Scholarship

$2-1-2009$

\title{
Current-voltage Relation For A Field Ionizing He Beam Detector
}

\section{P. DePonte}

Physics Department, Arizona State University, Tempe, Arizona 85281, USA

Greg Elliott

University of Puget Sound, gelliott@pugetsound.edu

S.D. Kevan

Physics Department, University of Oregon, Eugene, Oregon 97403-1274, USA

Follow this and additional works at: http://soundideas.pugetsound.edu/faculty_pubs

\section{Citation}

Deponte, D. P., Gregory S. Elliott, and S. D. Kevan. 2009. "Current-voltage relation for a field ionizing He beam detector." Journal Of Applied Physics 105(4): 044910-044910.

This Article is brought to you for free and open access by the Faculty Scholarship at Sound Ideas. It has been accepted for inclusion in All Faculty Scholarship by an authorized administrator of Sound Ideas. For more information, please contact soundideas@pugetsound.edu. 


\title{
Current-voltage relation for a field ionizing He beam detector
}

\author{
D. P. DePonte, ${ }^{1, a)}$ Greg S. Elliott, ${ }^{2}$ and S. D. Kevan ${ }^{3}$ \\ ${ }^{1}$ Physics Department, Arizona State University, Tempe, Arizona 85281, USA \\ ${ }^{2}$ Physics Department, University of Puget Sound, Tacoma, Washington, D.C. 98416, USA \\ ${ }^{3}$ Physics Department, University of Oregon, Eugene, Oregon 97403-1274, USA
}

(Received 18 August 2008; accepted 11 January 2009; published online 25 February 2009)

\begin{abstract}
Emerging interest in utilizing the transverse coherence properties of thermal energy atomic and molecular beams motivates the development of ionization detectors with near unit detection efficiency and adequate spatial resolution to resolve interference fringes of submicron dimension. We demonstrate that a field ionization tip coupled to a charged particle detector meets these requirements. We have systematically studied the current-voltage relationship for field ionization of helium using tungsten tips in diffuse gas and in a supersonic helium beam. For all 16 tips used in this study, the dependence of ion current on voltage for tips of fixed radius was found to differ from that for tips held at constant surface electric field. A scaling analysis is presented to explain this difference. Ion current increased on average to the 2.8 power of voltage for a tip at fixed field and approximately fifth power of voltage for fixed radius for a liquid nitrogen cooled tip in room temperature helium gas. For the helium beam, ion current increased as 2.2 power of voltage with constant surface field. The capture region of the tips was found to be up to $0.1 \mu \mathrm{m}^{2}$ for diffuse gas and $0.02 \mu \mathrm{m}^{2}$ in the beam. Velocity dependence and orientation of tip to beam were also studied.

(C) 2009 American Institute of Physics. [DOI: 10.1063/1.3081641]
\end{abstract}

\section{INTRODUCTION}

Thermal energy helium atom beams having de Broglie wavelengths on the order of $0.1 \mathrm{~nm}$ have been widely applied to study surface structure and dynamical properties. ${ }^{1,2}$ Applications that extract the coherent fraction from such beams in one transverse dimension have been carried out, ${ }^{3-6}$ and recent transverse dimensional coherent experiments have been accomplished. ${ }^{7,8}$ Spatial filtering renders the coherent flux available in such experiments many orders of magnitude lower than the incoherent flux used in conventional helium scattering experiments. For this reason, these emerging applications motivate the development of detectors with near unit helium atom detection efficiency and adequate spatial resolution to resolve interference fringes of submicron dimension. ${ }^{9}$ A candidate design that satisfies these requirements consists of a field ionization (FI) tip coupled to a charged particle detector. A sharp tip at high voltage first polarizes and then binds nearby atoms and molecules until they field ionize, thereby satisfying the requirement of high ionization efficiency over a small capture region. ${ }^{10}$ FI detectors are also thought to have picosecond response times. The area of the capture region, and thus the spatial resolution of the detector, depends on the size of the tip, the applied field, and the properties of the gas source. Previously reported measurements of the capture region for an ambient gas near equilibrium and its dependence on tip parameters have led to divergent results. ${ }^{11-16}$ Moreover, just two measurements, ${ }^{9,17}$ for a directed, supersonic gas source have been reported and the dependence on tip parameters was not systematically investigated. This capture region in a supersonic beam is

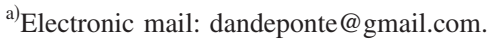

smaller than desired in some applications, and in any case one would like to understand and to control the size of capture region.

The capture region of an FI tip is highly dependent on tip shape, as is implied by the divergent results of previous studies. A tip radius can be estimated by Fowler-Nordheim equation, scanning electron microscopy (SEM) image, or field ion microscopy, but attempts to model capture region based on a single parameter, such as tip radius, are only very roughly quantitative. Such a model does not even allow for consistent qualitative prediction; in our experience a smaller FI tip may not necessarily have smaller capture region than a larger radius tip. There has been considerable work over the last three decades on gas FI for focused ion beam, FIB, sources as recently reviewed by Tondare. ${ }^{18}$ While having some relevance to detector applications, it is intensity and brightness rather than overall ion current that is the desired for FIB application. Multiple detectors used as either two dimensional arrays ${ }^{19,20}$ or "activated" wires in which sharp filaments are grown or deposited on a surface $e^{21,22}$ have been used to produce high ion current for sample analysis but at the time of this writing activated surfaces have shown very little promise as high efficiency, high resolution field ion detectors, and two dimensional arrays would require considerable development.

The characteristics of $I-V$ curves from field ionizing tips in the presence of gases has been well studied and the physical mechanisms involved are well understood. ${ }^{10,23}$ Two voltage regions characterize a typical $I-V$ curve for a cold tip, as shown in Fig. 1: (1) above a threshold voltage there is initially a steep rise in the ion current as the tunneling of electrons from atom to tip turns on, and (2) above a "knee" voltage there is a less steep rise, where the ion current is flux limited rather than tunneling limited. We are particularly in- 


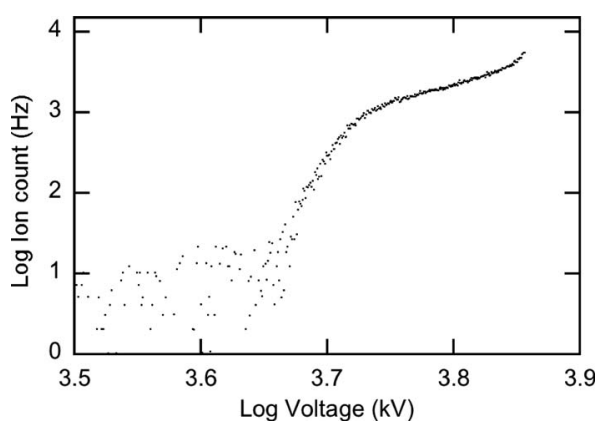

FIG. 1. $I-V$ characteristic for helium ionization from a $\mathrm{W}$ tip at $77 \mathrm{~K}$ showing distinct regions, reading from left to right: ionization of background gas, a steep rise at the onset of He ionization, a lower slope beyond the knee voltage and a rise at the far right due to $\mathrm{W}$ evaporation.

terested in the second of these regions since it allows us to vary the size of the capture region. For a tungsten tip at room temperature and approaching the knee voltage, the surface field at the tip of about $500 \mathrm{MV} / \mathrm{cm}$ is sufficient to cause tungsten to evaporate. ${ }^{24}$ Colder tips have a higher evaporation field $540 \mathrm{MV} / \mathrm{cm}$ at $77 \mathrm{~K} .{ }^{25}$ Thus, for room temperature tungsten tips used to ionize helium, only the first region of the $I-V$ curve is observable without altering the shape of the tip.

\section{EXPERIMENTAL PROCEEDURE}

\section{A. FI Tip preparation}

FI tips can be made by a several methods. ${ }^{26-30}$ We chose to electrochemically etch 200 micron $\mathrm{W}$ wire in a $0.5 \mathrm{M}$ solution of $\mathrm{NaOH}$ using a few volts $60 \mathrm{~Hz}$ ac. The sharpness of the tip depends on concentration, length of wire, time in solution, and current. The etching process produces a conical shape to the wire below the surface of the solution. The cone shortens as the etching proceeds; in general, longer cones have sharper ends than shorter cones, ${ }^{28}$ and etching at a faster rate produces sharper tips than etching at slower rates. FI tips made by electrochemical etching did not have any observable limit to shelf life. FI tips that were left at atmosphere for months were still able to ionize helium when installed in the detector. An oxide layer, ${ }^{31}$ as well as other condensates which form on the tungsten over time can be removed as the tip is brought up to high voltage. The FI tips were not annealed, which in the absence of an applied field will produce a spherical tip due to surface tension, ${ }^{29,32}$ but a conical tip is necessary for field evaporation.

FI tips with a rough or fractured surface were also tried. The dc voltage lamella drop off technique produces tips of short shank and rough surface as desired for scanning tunnel microscope tips. ${ }^{33}$ The rough surface of the broken wire can be used to produce ions and has been employed in the past for field desorption and ionization. ${ }^{30}$ When used for FI, we found ion current from such tips fluctuates rapidly in time, making the tip unsuitable as an ionization detector. A tip that is operating properly will appear to show only shot noise while a rough surface can produce an ion current that occasional fluctuates by a factor of 2 .

Occasionally a good tip will fail during evaporation but still be able to produce ions if the voltage is increased fur- ther. This can happen if the tip arcs, melting and reforming the tip at larger radius. Dyke and Dolan ${ }^{27}$ have reported using "vacuum arcing" to increase the size of their field emission tips, but we find the resulting field ion current too unstable for our use. Our tips are made from polycrystalline wire and can fail by breaking at grain boundaries. Polycrystalline wire contains crystallites tens of nanometers wide which can be removed by the field. ${ }^{31}$

\section{B. FI tip testing}

After electrochemical etching, the tips were rinsed in distilled water and air dried. For FI testing, each tip was individually mounted on the end of a high voltage vacuum feedthrough in front of a channel electron multiplier (CEM). To prevent secondary electrons from being drawn away from the multiplier, a grounded spherical wire screen was placed between the tip and the multiplier cone $1 \mathrm{~cm}$ away from the tip. The tip could be cooled to $80 \mathrm{~K}$ via heat conduction through the center conductor of the high voltage vacuum feedthrough to a liquid nitrogen reservoir outside the vacuum. For FI measurements, the LABVIEW data acquisition program was used both to control the voltage on the tip and to measure the ionization count rate. The tip voltage was incremented in $10 \mathrm{~V}$ steps with an adjustable dwell time on each voltage step, ranging from $2 \mathrm{~s}$ for smaller tips at lower count rates to $300 \mathrm{~ms}$ for larger tips at higher count rates. The output pulses from the CEM were processed by a digital amplifier discriminator, and a count rate was calculated from the number of pulses within a narrow time window, typically 10-100 ms.

Diffuse gas ionization experiments were carried out in a turbo-molecular pumped ultrahigh vacuum test chamber with a base pressure of $3 \times 10^{-10}$ mbar. A background pressure of $1.3 \times 10^{-8}$ mbar of helium was used for all diffuse gas ionization rate measurements. It has been shown ${ }^{34-36}$ that the ion current from a field ionizing tip is greatest from "hot spots" on the tip surface that may change over time, and that the ion current may be distributed off the axis of the tip. Tips were first tested in an apparatus in which the tip could be moved laterally in front of a CEM, to test if the angular distribution of ions was significantly skewed away from the tip axis. Any tips that emitted ions noticeably off axis were discarded. Angular distribution also changes with field, becoming wider with increasing field strength. ${ }^{37}$ It is therefore important to use a multiplier with a large opening to collect as much of the ion current as possible. The ends of the tips were held in the plane of a $3 \mathrm{~cm}$ diameter multiplier cone opening, allowing for current collection over a full hemisphere. As the ionization threshold voltage is sensitive both to the tip-to-screen distance and shape of the tip holder, a fixture was used to repeatedly position the tip to within $1 \mathrm{~mm}$ of the plane of the multiplier cone opening, and to within 2 $\mathrm{mm}$ of the center of the cone.

The helium beam ionization experiments were performed in an ion-pumped ultrahigh vacuum system with a base pressure of $2 \times 10^{-10}$ mbar. A sketch of the apparatus is given in Fig. 2. The helium beam was generated using a ten micron steel nozzle, and collimated first by a skimmer and 

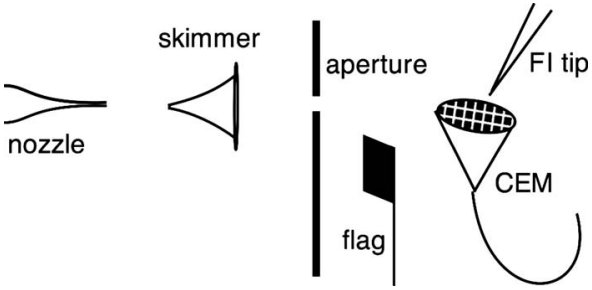

FIG. 2. A schematic of the supersonic beam apparatus showing the beam nozzle, the skimmer (which serves as a first vacuum barrier), and a $200 \mu \mathrm{m}$ aperture (which serves as a second vacuum barrier). In the detection chamber is a movable flag to block the beam, and a FI tip in front of a CEM covered by a grounded screen.

then by a $200 \mu \mathrm{m}$ aperture, which both serve as vacuum barriers between chambers. Due to space limitations a multiplier with a smaller, one-centimeter, opening was used. The tip was held a few millimeters away from a grounded screen covering the multiplier. Tips were welded to a small, $50 \mathrm{~mm}^{3}$ steel mount that was thermally and electrically insulated from the linear motion stage to which it was attached. The mount was cooled via conduction through the high voltage feedthrough to a liquid nitrogen reservoir.

Starting with a newly prepared tip, a set of $I-V$ scans was taken following incremental field evaporation of the FI tip. For the first scan, the tip voltage was raised until a helium signal was observed, and then held steady until field evaporation ceased and the ion current stabilized. $I-V$ scans were taken by decreasing the voltage from this value, and scans taken in this fashion were repeatable and showed no hysteresis. All diffuse gas $I-V$ scans were done both with and without helium, so that contribution to the ion count from the UHV background gases could be subtracted. For the helium beam measurements, a movable flag was used to block the beam inside the UHV chamber. After each scan was taken in the beam, another scan would be taken with the beam blocked by the flag so that diffuse background gas contribution to the ion current could be recorded. After a repeatable $I-V$ scan was acquired, the tip voltage was slowly raised by field evaporation to a new maximum value, typically $20 \%$ higher, to create a tip of larger radius. Another scan would be taken, and the process repeated until the tip failed. For some tips, this process was successfully repeated five to ten times before the tip failed. The majority of tips either failed on the first cycle or only survived a few cycles before failing. We report here a data set collected from three tips at room temperature and eleven tips at $80 \mathrm{~K}$.

\section{RESULTS}

\section{A. FI tips in a diffuse gas}

A set of nine $I-V$ curves is shown in Fig. 3(a), measured from a single FI tip immersed in a diffuse helium gas that underwent successive field evaporation to higher operating voltages. The curves are plotted on a log-log scale and labeled by their highest operating voltage, which vary from 2.2 up to $7.2 \mathrm{kV}$. Each individual $I-V$ curve gives the dependence of ion current on surface field at constant tip radius. The upturn at the highest voltages on some of these curves is due to field evaporation, which could be eliminated by starting
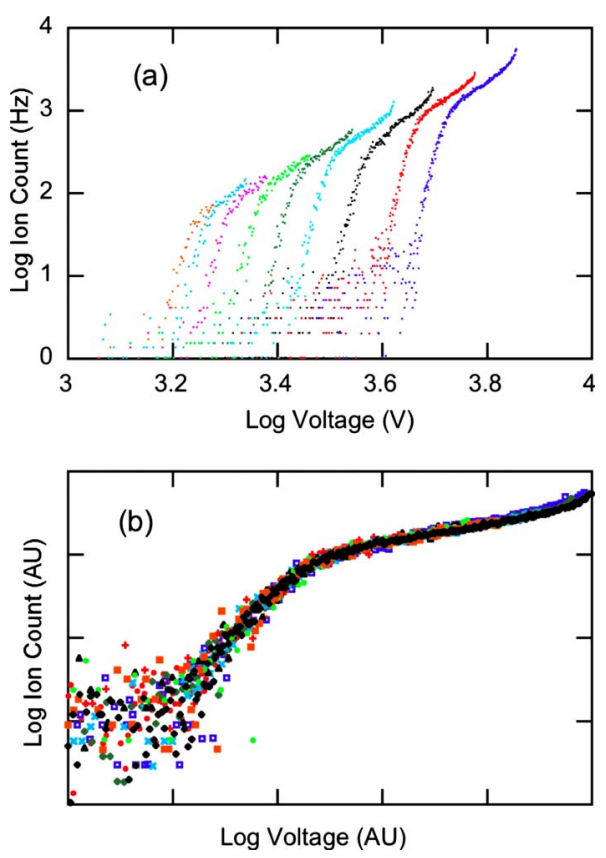

FIG. 3. (Color online) Shown in (a) is a set of nine $I-V$ scans from a single tip taken after successive field evaporation from 2.2 to $7.2 \mathrm{kV}$ and in (b) the same data with all scans scaled to a single $I-V$ curve. The mapping to a single curve generates a set of $\left(C_{I}, C_{V}\right)$ scaling pairs, shown in Fig. 5.

the $I-V$ scan a few hundred volts below the maximum voltage. The scans all exhibit the basic behavior described previously: an ionization threshold voltage, followed by a steep increase, followed by a less steep increase past what we call the "knee" voltage. Above the knee voltage, in the fluxlimited regime, the ion current may be approximated as the power law relationship

\section{$I \propto V^{\gamma} \quad$ (constant tip radius)}

with an average $\gamma$ between 4.5 and 5.5. The exponent $\gamma$ is dependent on tip shape ${ }^{11}$ and tip temperature ${ }^{13}$ and is not constant, but decreases with increasing voltage above the knee. There is also some variation in $\gamma$ from one $I-V$ scan to another.

The change in the $I-V$ curves following successive field evaporation show the effect of tip radius on the $I-V$ characteristics. As the highest operating voltage is increased, field evaporation increases the radius of curvature at the tip. This can be seen in Fig. 4, which shows two SEM images of the same tip before and after field evaporation. Field evaporation removes the greatest material from where the radius of curvature is smallest, and where the surface field is the largest.

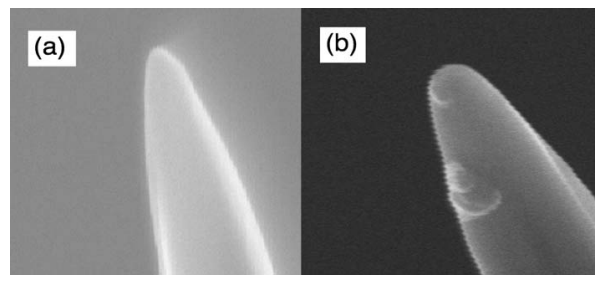

FIG. 4. SEM pictures of a tip before and after field evaporation showing an increase in the tip radius. In (a) the maximum tip voltage was $10 \mathrm{kV}$; in (b) the maximum tip voltage was increased to $15 \mathrm{kV}$. 


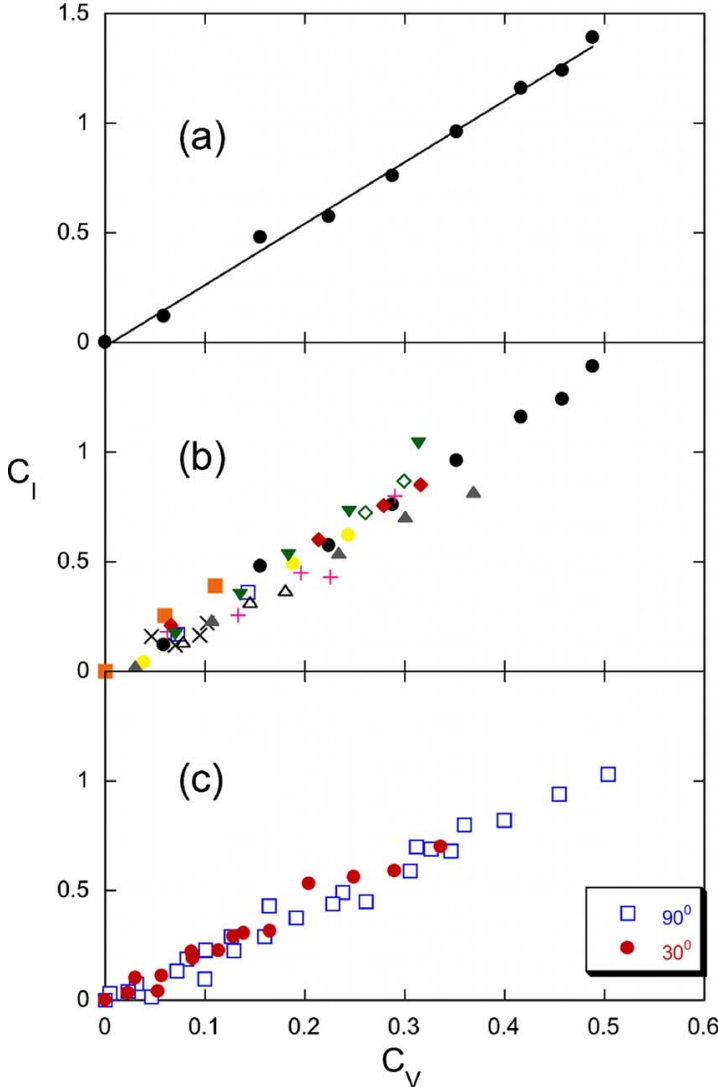

FIG. 5. (Color online) Shown in (a) are the nine $\left(C_{I}, C_{V}\right)$ scaling pairs for the data shown in Fig. 3, with the slope of the best fit line determining the exponent beta. Data for all tips are shown for (b) static gas and (c) supersonic helium beam, at $30^{\circ}$ and $90^{\circ}$ to the tip.

At the very end of the tip, the shape is nearly spherical, and the radius has clearly increased upon field evaporation. Away from the end of the tip, in the "shank" region, the shape of the tips are different, hence the tip before and after field evaporation deviates from geometrical similarity the further one looks away from the end of the tip. The dominant effect on the $I-V$ curves results from the increase in tip radius. Examining the $I-V$ curves in Fig. 3(a), for the larger size tips it takes a higher voltage to achieve the same surface electric field, and hence the threshold and knee voltages shift to higher values. The ion current at the knee voltage also increases.

All the $I-V$ curves are similar in that they can be made to lie on top of one another by a shift along each axis, with the mapping,

$$
\begin{aligned}
& \log \left(I^{\prime}\right)=\log (I)+c_{I}, \\
& \log \left(V^{\prime}\right)=\log (V)+c_{v} .
\end{aligned}
$$

All curves in Fig. 3(a) have been shifted in this manner to lie on top of one another as shown in Fig. 3(b). The pairs $\left(c_{I}, c_{V}\right)$ used to scale each scan in Fig. 3 have been plotted in Fig. 5 (a) and the slope of the resulting line, $\beta$, is used to determine the current-voltage relationship at constant field
TABLE I. Exponent beta values for tungsten FI tips at $77 \mathrm{~K}$ in diffuse helium gas. The third column is the highest voltage to which the tip was field evaporated without failure, and the fourth column is the ratio of the highest evaporation voltage to the voltage at which field evaporation was first observed. Also included are beta values for two warm tips, as well as data from other studies.

\begin{tabular}{cccc}
\hline \hline$\beta$ & Number of data points & $\begin{array}{c}V_{\max } \\
(\mathrm{kV})\end{array}$ & $V_{\max } / V_{\min }$ \\
\hline $2.85 \pm 0.06^{\mathrm{a}}$ & 9 & 7.2 & 3.8 \\
$2.5 \pm 0.3$ & 3 & 14.5 & 1.5 \\
$2.9 \pm 0.1$ & 3 & 25 & 1.9 \\
$2.0 \pm 0.3$ & 5 & 33 & 1.5 \\
$2.4 \pm 0.1$ & 6 & 7.2 & 2.4 \\
$2.1 \pm 0.2$ & 4 & 26 & 1.7 \\
$2.5 \pm 0.2$ & 4 & 25 & 1.9 \\
$3.4 \pm 0.4$ & 3 & 18 & 1.5 \\
$2.7 \pm 0.1$ & 5 & 12.5 & 2.5 \\
$2.3 \pm 0.1$ & 6 & 18 & 2.5 \\
$3.5 \pm 0.1$ & 6 & 12.5 & 2.5 \\
$2.5 \pm 0.1^{\mathrm{b}}$ & 9 & 13 & 2.9 \\
$2.3 \pm 0.3^{\mathrm{b}}$ & 7 & 15 & 1.5 \\
$2.3 \pm 2.9^{\mathrm{c}}$ & 5 & & 1.3 \\
$2.5-2.8^{\mathrm{d}}$ & 5 & & 1.7 \\
$2.5-3^{\mathrm{e}}$ & 5 & & 2.5 \\
\hline \hline
\end{tabular}

These values used for Fig. 5 .

${ }^{\mathrm{b}}$ Warm tips

${ }^{\mathrm{c}}$ Reference $42,\langle 111\rangle$ plane

${ }^{\mathrm{d}}$ Reference 42, $\langle 011\rangle$ plane

${ }^{\mathrm{e}}$ Reference 11 .

$I \propto V^{\beta} \quad($ constant surface field $)$.

Relations (2) and (3) have held up with the same degree of agreement as in Fig. 3(b) for every tip that survived the process of successive field evaporation to produce a set of $I-V$ curves, but there is a large spread in $\beta$ value obtained for each of the 11 different tips. In Fig. 5(b), the knee coordinates for eleven such tips are plotted versus tip voltage, and in Table I a summary of the data sets for these eleven tips is presented. The number of successive field evaporation cycles for these eleven tips vary from two up to ten. The scaling parameter $\beta$ varies from 2 up to 3.5, with an average of 2.8, somewhat higher than $\beta=2$ predicted by Muller. ${ }^{29}$ Two room temperature tips were also studied to determine $\beta$, shown in Table I. Even though these warm tips had very little working range, and $\gamma$ values could be determined, there was still sufficient structure to permit these scans to be scaled to a universal curve with a high degree of accuracy.

While relations (2) and (3) hold for each tip, no such relation was found when comparing different tips. Two tips with the same highest operating voltage may produce ion currents which differ by an order of magnitude if one was chemically etched and the other first etched to a smaller radius then field evaporated to its final size. This may be related to variation in tip shape during evaporation or etching as explained in Sec. IV.

The capture region for FI tips in diffuse gas is determined ${ }^{9}$ from the measured ion count rate. With no field and assuming a Maxwell-Boltzmann gas at temperature $T$ 
TABLE II. Exponent beta values for directed helium flow, for two different angles between beam and tip. The fourth column is the highest voltage to which the tip was field evaporated without failure and the fifth column is the ratio of the highest evaporation voltage to the voltage at which field evaporation was first observed.

\begin{tabular}{ccccc}
\hline \hline Angle & Beta & Number of data points & $\begin{array}{l}V_{\max } \\
(\mathrm{kV})\end{array}$ & $V_{\max } / V_{\min }$ \\
\hline $30^{\circ}$ & $2.2 \pm 0.2$ & 7 & 11 & 1.8 \\
$30^{\circ}$ & $2.1 \pm 0.2$ & 10 & 15.5 & 2.4 \\
$90^{\circ}$ & $2.2 \pm 0.3$ & 4 & 12 & 1.5 \\
$90^{\circ}$ & $2.1 \pm 0.1$ & 8 & 9 & 2.7 \\
$90^{\circ}$ & $2.3 \pm 0.3$ & 5 & 14 & 1.4 \\
$90^{\circ}$ & $2.1 \pm 0.1$ & 15 & 19 & 3.8 \\
\hline \hline
\end{tabular}

and pressure $P, \dot{N}$ atoms per second will pass through a spherical surface whose cross sectional area is given by

$$
\sigma=\frac{\dot{N}}{P} \sqrt{\frac{\pi m k T}{8}}
$$

with $m$ the mass of a helium atom. For a FI tip, if the assumption is made that any atom entering the capture region is ionized, $\sigma$ may then calculated using $\dot{N}$ to be the measured ion count rate. The largest values for count rate, that closest to the evaporation voltage, was used to calculate $\sigma$ for all cold tips. The values of $\sigma$ in our diffuse gas setup for a $77 \mathrm{~K}$ tip in $300 \mathrm{~K}$ helium ranged from 0.1 to $0.001 \mu \mathrm{m}^{2}$ depending on voltage before and after evaporation. There have been many other reports on current or count rate versus pressure for diffuse gas ${ }^{11-16}$ which using Eq. (4) range in value of $\sigma$ from a few $\mu \mathrm{m}^{2}$ to a few hundredths $\mu \mathrm{m}^{2}$ depending on gas and tip temperature and tip size. In older reports there is no clear trend in $\sigma$ with tip size or temperature from one report to the next. It was demonstrated ${ }^{11}$ that difficulty in accounting for secondary electrons may have lead to some of this large range in $\sigma$ however variation in tip shape is also a contributing factor.

\section{B. FI tips in a supersonic helium beam}

The above procedure was repeated for six tips using a supersonic helium beam. We found $\gamma$ between 7.6 and 8.2 for four tips in the beam; we were unable to suitably fit data with a straight line for the two remaining tips. It has been reported $^{16}$ that the size of the capture region around a tip depends on the angle that the tip makes with the beam. We examined tips at $30^{\circ}$ to the beam axis and perpendicular to the beam axis as a means to decouple the ionization and capture region effect on $\beta$ but negligible difference was found. Figure $5(\mathrm{c})$ shows the $\left(C_{I}, C_{V}\right)$ pairs for all six tips used in the beam labeled by orientation. All values of $\beta$ are shown in Table II. The average values for $\beta$ at $30^{\circ}$ and at $90^{\circ}$ are both 2.2 .

As in Sec. III A, all scans were similar in shape. However the universal curve for the beam appears different from that for static gas. The tips in the static gas have a more distinct knee, a larger working range, and a smaller $\gamma$, all of which indicate a lower tip temperature. The tips in the diffuse gas setup had a shorter thermal conduction path to the liquid nitrogen reservoir and may have been colder than the tips in the beam setup. It is also likely that the smaller multiplier opening used for the beam measurements resulted in an underestimation of $\beta$.

There have been three reports on $\sigma$ for effusive sources $^{16,38,39}$ and only two for a supersonic source. ${ }^{9,17}$ A 1966 report on an effusive beam ${ }^{16}$ [at $300 \mathrm{~K}$ (Ref. 9)] had $\sigma$ of order $1 \mu^{2}$ for $\mathrm{He}, \mathrm{Ar}$, and $\mathrm{N}_{2}$. That report cites another report of the same author of $10^{-6} \mathrm{Amp} /$ torr sensitivity for $\mathrm{Ar}$ and $\mathrm{N}_{2}$ diffuse gas, or $\sigma=0.1 \mu \mathrm{m}^{2}$ using Eq. (4). The report on an effusive source by Woods and Fenn ${ }^{38}$ demonstrated the utility of FI as a detector but contains no quantitative information on ion current. In 1974 Mcwane and Oates $^{39}$ reported $\sigma=0.2 \mu \mathrm{m}^{2}$, independent of tip size or velocity, for an effusive He beam between 0.3 and 4 K. Doak ${ }^{9}$ has recently revisited this paper and cast doubt on the velocity result, reporting $\sigma=5 \times 10^{-5} \mu \mathrm{m}^{2}$ for a $300 \mathrm{~K}$ beam and $80 \mathrm{~K}$ tip. As for independence on tip size, it was suggested by Mcwane and Oates that diffusion may be responsible. It was demonstrated earlier by Halpern and Gomer ${ }^{40}$ that diffusion from shank to the tip dominates supply at $4.3 \mathrm{~K}$, but it is unclear what tip temperature was used by Mcwane and Oates. A recent paper by Piskur et al. ${ }^{17}$ report $\sigma=9.5$ $\times 10^{-6}$ and $\sigma=1.05 \times 10^{-4} \mu \mathrm{m}^{2}$ for a supersonic beam at 298 and $77 \mathrm{~K}$, respectively, using a room temperature tungsten tip.

For our helium beam data, $\sigma$ is determined from beam intensity $I$ and the ion count rate;

$$
\sigma=\frac{\dot{N}}{I} d^{2},
$$

where $d$ is the nozzle to detector distance. The values of $\sigma$ in our beam setup are from $3.9 \times 10^{-5}$ to $6.2 \times 10^{-4} \mu \mathrm{m}^{2}$ before evaporation and from $6.2 \times 10^{-4}$ to $1.6 \times 10^{-3} \mu \mathrm{m}^{2}$ after evaporation for a room temperature beam. This capture region is $\approx 100 \times$ smaller than that found in the diffuse gas setup. The detector collected ions in an approximately $30^{\circ}$ half angle from the tip axis which is expected to result in an underestimation of $\sigma$ by about $20 \%$, far less than the measured difference.

The measured background consisted of helium ions from the diffuse background, nonhelium ion counts and noise from the ion pump in the detector chamber. Figure 6 shows measured signal with the FI tip in the direct beam, the background count with the flag blocking the beam just before the detector and the difference between the two. Both the FI tip and the ion pump produce an ion current that is related to the total background gas making it impossible to decouple the ion pump and FI tip background. We are therefore unable to directly compare $\sigma$ for static gas and supersonic jet.

The detection area of FI tips is velocity dependent. We routinely use FI detectors in our helium beam apparatus and have observed an increase in detection area as the helium beam is cooled. Preliminary data for a liquid nitrogen cooled tip at $71^{\circ}$ angle to the beam show a factor of 7 difference in detection area when used with a $300 \mathrm{~K}$ beam compared to a $77 \mathrm{~K}$ beam. This is not inconsistent with temperature dependence previously reported for diffuse gas. ${ }^{41}$ 


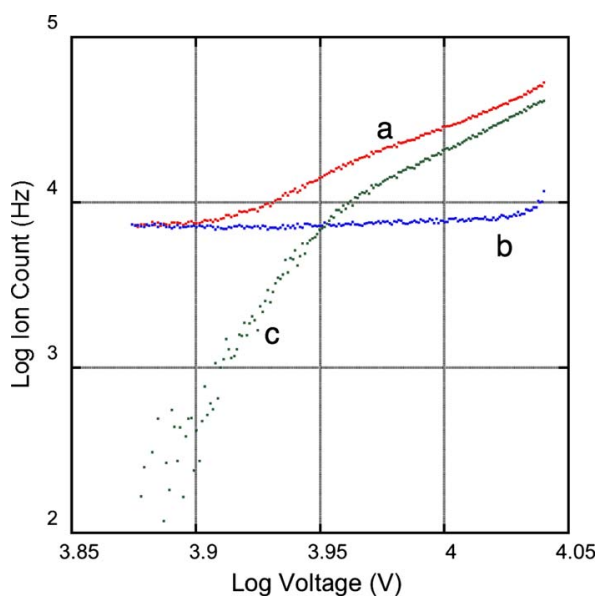

FIG. 6. (Color online) Measured ion count rate (a) with the FI tip in the direct beam, (b) with the beam blocked just before the detector, and (c) the difference giving the direct beam signal only.

\section{GEOMETRIC SCALING}

A consideration of geometric scaling sheds some light on the mapping of the $I-V$ curves onto a universal curve, and on the relative magnitudes of the exponents $\gamma$ and $\beta$, which characterize the dependence of the ionization current on voltage at constant size and constant surface field, respectively. We consider the effect of increased size alone for geometrically similar tips and then briefly discuss the effect of simultaneous changes in shape as well as size.

In the flux-limited regime above the voltage corresponding to the knee of an $I-V$ graph, we assume all of the flux through some surface surrounding the tip to be ionized. For simplicity we define this surface to enclose a volume of space around the tip that is above a critical threshold ionization field value. This model shows that an increase in the voltage on the tip results in an increase in the ion current through two effects: (1) the region above a critical threshold field increases in size and surface area, and (2) the atomic current density on the surface of this region increases. The geometric scaling of the first effect can be determined solely through consideration of the field outside of a tip. The scaling of the second effect can be made within a classical treatment of atom trajectories via Liouville's theorem.

A solid conductor of arbitrary shape held at a constant potential $V_{0}$ produces an electrostatic potential outside the conductor at location $\boldsymbol{r}$ that can always be expressed in the form $V(\boldsymbol{r}) / V_{0}=\tilde{V}(\boldsymbol{r} / d)$, where $d$ is a characteristic size of the conductor. The dimensionless function $\tilde{V}(\widetilde{\boldsymbol{r}})$, where $\widetilde{\boldsymbol{r}} \equiv \boldsymbol{r} / d$, can be thought of as the solution to the electrostatic problem for a conductor of similar shape but with unit size and unit applied potential. A neutral, polarizable atom in such an electrostatic field will feel a polarization potential that to lowest order is given by $\mathcal{U}(\boldsymbol{r})=\alpha|\nabla V|^{2}$, where $\alpha$ is the atomic polarizability. This polarization potential can also be expressed in the form $U(\boldsymbol{r}) / U_{0}=\tilde{U}(\widetilde{\boldsymbol{r}})$, where $U_{0}=\alpha\left(V_{0} / d\right)^{2}$. Thus if two tips of the same shape have the same ratio $V_{0} / d$, they have geometrically similar field distributions that map onto the same dimensionless field $\tilde{U}(\widetilde{\boldsymbol{r}})$. This scaling is evident in the increase in the threshold voltage for ionization for tips that have increased their size due to field evaporation.

The classical equations of motion for the trajectory of an atom in this potential field can be similarly nondimensionalized using the tip length scale $d$ and a time scale $\tau$ $=\sqrt{m d^{2} / k_{B} T}$ generated from the thermal energy scale, where $m$ is the atom mass, $k_{B}$ is Boltzmann's constant, and $T$ is the gas temperature. Thus in the problem of two tips of the same shape but of different size, both the potential function and the equations of motion can be scaled to the same dimensionless problem. The family of solutions to the equations of motion, corresponding to different initial conditions, are therefore geometrically similar for two geometrically similar tips with the same ratio $V_{0} / d$. The scaling of the $I-V$ curves onto a single curve for tips undergoing successive evaporation is consistent with this geometric scaling and indicates that the predominant effect of the field evaporation is to increase the radius at the end of the tip.

The flux of neutral atoms delivered to the tip can be expressed in terms of a phase space distribution function $f(\boldsymbol{r}, \boldsymbol{v})$, and the current $I$ delivered to the surface can be calculated by integrating this flux over the surface.

$$
I=\int_{S} d S \int d^{3} \boldsymbol{v} f(\boldsymbol{r}, \boldsymbol{v}) \hat{n} \cdot \boldsymbol{v},
$$

where $S$ is as defined above and $\hat{\boldsymbol{n}}$ is a unit vector pointing outward from the surface $S$ at the location $\boldsymbol{r}_{s}$. For the diffuse gas experiments, the background pressure is low enough that the atoms are accelerated toward the tip in the absence of collisions with other atoms. In this case, the phase space distribution function $f$ on the surface $S$ can be simply related to the Maxwell-Boltzmann distribution function $f_{0}$ of the background gas via Liouville's theorem,

$$
f\left(\boldsymbol{r}_{s}, \boldsymbol{v}_{s}\right)=f_{0}\left(\boldsymbol{v}_{0}\right)=f_{0}\left(\boldsymbol{v}_{s}-\Delta \boldsymbol{v}\right),
$$

with $\Delta \boldsymbol{v} \equiv \boldsymbol{v}_{s}-\boldsymbol{v}_{0}$ the change between the far-field velocity $\boldsymbol{v}_{0}$ and the velocity $\boldsymbol{v}_{s}$ on the surface $S$. For a field sufficient to ionize helium, the energy of the atoms as they strike the tip is on the order of $1 \mathrm{eV}$. The integral in Eq. (6) can be approximated under the assumption that the atoms pick up most of their final energy in the region of high field very near the tip, and that therefore the atoms pass through $S$ nearly normally. This approximation decouples the velocity and the surface integrals in Eq. (6), and yields

$$
I \approx I_{\text {thermal }}+n_{0} v_{f} S(d, V),
$$

where the function $S(d, V)$ represents the area of a surface at a constant electric field magnitude outside a tip of size $d$ and at voltage $V, I_{\text {thermal }}$ is the current to the surface $S$ that would be present without the field, $n_{0}$ is the background gas density, and $\boldsymbol{v}_{f}$ is the atom speed at the surface $S$.

This simple model for the flux delivered to the tip provides an explanation for the variation in the current with voltage at constant size and at constant surface field, and why the exponent $\beta$ is less than the exponent $\gamma$. Above the knee the second term in Eq. (8) is much greater than the first term since the final velocity at the tip is much greater than the average thermal velocity. To good approximation the fi- 
nal velocity is, via conservation of energy, proportional to the surface field, whereas the surface area $S(d, V)$ is proportional to the area $d^{2}$. To investigate how the area function $S(d, V)$ depends on voltage we have calculated the field distribution for a tip in the shape of a prolate spheroid, for which an analytic result can be simply derived. From this field distribution we calculated $S(d, V)$ over a range of operating voltages and found $S(d, V)$ to vary approximately as a power law in the surface field. With the assumption that the area function for our experimental tips similarly scale as $S(d, V) \propto d^{2}(V / d)^{\delta}$, for some exponent $\delta$, the additional flux to the surface scales as

$$
v_{f} S(d, V) \propto \frac{V}{d} d^{2}(V / d)^{\delta}=d^{1-\delta} V^{1+\delta} .
$$

Relation (9) predicts that for tips held at constant size, the dominant current $n_{0} v_{f} S$ in Eq. (8) varies as voltage to the exponent $\gamma=1+\delta$, and for tips of different size at the same value of $V / d$, the current varies as voltage to the exponent $\beta=2$. The surface area calculations for the prolate spheroidal tips predict a range of values for the exponent $\delta$ from two to ten depending on the ellipticity of the spheroid, with our value $\gamma \approx 4.5$ consistent with a needlelike shaped tip. However, our actual tip shapes are not spheroidal, and a further comparison with that shape model would not be useful.

We measure a range of values for the exponent $\beta$, consistently larger than two, the value predicted by our flux model for tips of the same shape but different size. A larger value for $\beta$ would be consistent with certain changes in shape as well as size during field evaporation, as in evident in Fig. 4. If one compares a tip with a radius of curvature $R$ and a sphere with the same radius, it is well known that more voltage per unit radius is required to achieve the same surface field at the end of the tip as for the sphere. ${ }^{10}$ This factor can be simply calculated given a model for the tip shape; for tips in the shape of prolate spheroids, the factor increases sharply as the shape progresses from nearly spherical to more needlelike. Beta values larger than two are consistent with the flux model if field evaporation increases the voltage per unit radius needed to achieve a fixed field. The scatter in the measured $\beta$ values can be explained by variations in the rate of change in the critical voltage per unit radius with the shape changes induced by field evaporation.

\section{DISCUSSION}

Field evaporation produces tips whose $I$ - $V$ characteristics are similar in shape and obey simple scaling consistent with the geometric scaling model of Sec. IV. For each tip examined, ion current increased as $V^{\gamma}$ above the knee voltage and as $V^{\beta}$ for successive evaporation. No such scaling was found when comparing tips prepared from different tungsten wires. It is likely that chemical etching produces a different evolution of tip shape than does field evaporation. In all cases $\gamma>\beta$; ion current increases more rapidly with voltage at constant tip size than compared to that at constant surface field. This implies that at any voltage greater than the knee voltage, more ions are made by a small FI tip than by a large FI tip. Therefore, in applications where the largest possible capture region is desired, a small tip operating well above the knee of the $I-V$ curve is strongly desired. By contrast, if a small capture region is desired, operating near the knee between regions I and II is desired.

In our current coherent atom beam measurements, ${ }^{8}$ the ion current for the tips in the helium beam is disappointingly small, implying a smaller than desired detection area. The ideal detection area for dynamic atom scattering of order $1 \mu \mathrm{m}^{2}$ is three orders of magnitude larger than the ionization cross section reported here as derived from the ion current in Sec. III A. The majority of this deficit may be met by working at higher voltage or using a gas that is more easily polarized and ionized. Finding a tip material with a higher evaporation field would also be useful to exploit the relative steepness, $\gamma$, of the $I-V$ curves compared to $\beta$. For atom beam microscopy a two dimensional array of such detectors would be ideal but requires considerable development from the current state of the art.

\section{ACKNOWLEDGMENTS}

This work was carried out at the University of Oregon with financial support provided by the National Science Foundation under Grant No. DMR-0506241 and the Department of Education GAANN Fellowship P200A010820.

${ }^{1}$ D. Farias and K.-H. Rieder, Rep. Prog. Phys. 61, 1575 (1998).

${ }^{2}$ Helium Atom Scattering from Surfaces, edited by E. Hulpke (SpringerVerlag, Berlin, 1992).

${ }^{3}$ A. Kalinin, O. Kornilov, L. Rusin, J. P. Toennies, and G. Vladimirov, Phys. Rev. Lett. 93, 163402 (2004).

${ }^{4}$ W. Schollkopf and J. P. Toennies, Science 266, 1345 (1994).

${ }^{5}$ M. Arndt, O. Nairz, J. Vos-Andreae, C. Keller, G. van der Zouw, and A. Zeilinger, Nature (London) 401, 680 (1999).

${ }^{6}$ M. S. Chapman, C. R. Ekstrom, T. D. Hammond, R. A. Rubenstein, J. r. Schmiedmayer, S. Wehinger, and D. E. Pritchard, Phys. Rev. Lett. 74, 4783 (1995)

${ }^{7}$ S. R. G. S. T. R. G. B. W. E. E. B. H. M. Koch, J. Microsc. 229, 1 (2008). ${ }^{8}$ F. S. Patton, D. P. Deponte, G. S. Elliott, and S. D. Kevan, Phys. Rev. Lett. 97, 013202 (2006).

${ }^{9}$ R. B. Doak, Y. Ekinci, B. Holst, J. P. Toennies, T. Al-Kassab, and A. Heinrich, Rev. Sci. Instrum. 75, 405 (2004).

${ }^{10}$ R. Gomer, Field Emission and Field Ionization (Harvard University Press, Cambridge, 1961).

${ }^{11}$ T. T. Tsong and E. W. Muller, J. Appl. Phys. 37, 3065 (1966).

${ }^{12}$ U. Feldman and R. Gomer, J. Appl. Phys. 37, 2380 (1966).

${ }^{13}$ M. J. Southon and D. G. Brandon, Philos. Mag. 8, 579 (1963).

${ }^{14}$ B. J. Waclawski and E. W. Muller, J. Appl. Phys. 32, 1472 (1961).

${ }^{15}$ E. W. Muller and K. Bahadur, Phys. Rev. 102, 624 (1956).

${ }^{16}$ W. D. J. Johnston and J. G. King, Rev. Sci. Instrum. 37, 475 (1966).

${ }^{17}$ J. Piskur, L. Borg, A. Stupnik, M. Leisch, W. E. Ernst, and B. Holst, Appl. Surf. Sci. 254, 4365 (2008).

${ }^{18}$ V. N. Tondare, J. Vac. Sci. Technol. A 23, 1498 (2005).

${ }^{19}$ C. A. Spindt, J. Appl. Phys. 39, 3504 (1968).

${ }^{20}$ C. A. Spindt, Surf. Sci. 266, 145 (1992).

${ }^{21}$ D. J. Riley, Mark Mann, D. A. MacLaren, P. C. Dastoor, and W. Allison, Nano Lett. 3, 1455 (2003).

${ }^{22}$ H. D. Beckey, J. Phys. E 12, 72 (1979).

${ }^{23}$ E. W. Muller, in Advances in Electronics and Electron Physics, edited by L. Marton (Academic, New York, 1960), Vol. 13, pp. 88-179.

${ }^{24}$ E. W. Muller, Phys. Rev. 102, 618 (1956).

${ }^{25}$ E. W. Muller and R. D. Young, J. Appl. Phys. 32, 2425 (1961).

${ }^{26}$ A. J. Melmed, J. Vac. Sci. Technol. B 9, 601 (1991).

${ }^{27}$ W. P. Dyke and W. W. Dolan, Adv. Electron. Electron Phys. 8, 89 (1956).

${ }^{28}$ W. P. Dyke, J. K. Trolan, W. W. Dolan, and G. Barnes, J. Appl. Phys. 24, 570 (1953)

${ }^{29}$ E. W. Muller, in Advances in Electronics and Electron Physics, edited by L. Marton (Academic, New York, 1960), Vol. 13, pp. 83-177. 
${ }^{30}$ M. Anbar, Anal. Chem. 48, 198 (1976).

${ }^{31}$ A.-D. Muller, F. Muller, M. Hietschold, F. Demming, J. Jersch, and K. Dickmann, Rev. Sci. Instrum. 70, 3970 (1999).

${ }^{32}$ P. C. Bettler and F. M. Charbonnier, Phys. Rev. 119, 85 (1960).

${ }^{33}$ M. Klein and G. Schwitzgebel, Rev. Sci. Instrum. 68, 3099 (1997).

${ }^{34}$ G. R. Hanson and B. M. Siegel, J. Vac. Sci. Technol. 16, 1875 (1979).

${ }^{35}$ G. R. Hanson and B. M. Siegel, J. Vac. Sci. Technol. 19, 1176 (1981).

${ }^{36}$ P. R. Schwoebel and G. R. Hanson, J. Vac. Sci. Technol. B 3, 214 (1985)
${ }^{37}$ H. D. Beckey, Principles of Field Ionization and Field Desorption Mass Spectrometry (Pergamon, New York, 1977).

${ }^{38}$ R. O. Woods and J. B. Fenn, Rev. Sci. Instrum. 37, 917 (1966).

${ }^{39}$ J. W. McWane and D. E. Oates, Rev. Sci. Instrum. 45, 1145 (1974).

${ }^{40}$ B. Halpern and R. Gomer, J. Chem. Phys. 51, 5709 (1969).

${ }^{41}$ K. Jousten, K. Bohringer, and S. Kalbitzer, Appl. Phys. B: Photophys. Laser Chem. 46, 313 (1988).

${ }^{42}$ Y. C. Chen and D. N. Seidman, Surf. Sci. 27, 231 (1971). 
Journal of Applied Physics is copyrighted by the American Institute of Physics (AIP). Redistribution of journal material is subject to the AIP online journal license and/or AIP copyright. For more information, see http://ojps.aip.org/japo/japcr/jsp 